\title{
EDITORIAL
}

\section{Covering gastroenterology and hepatology}
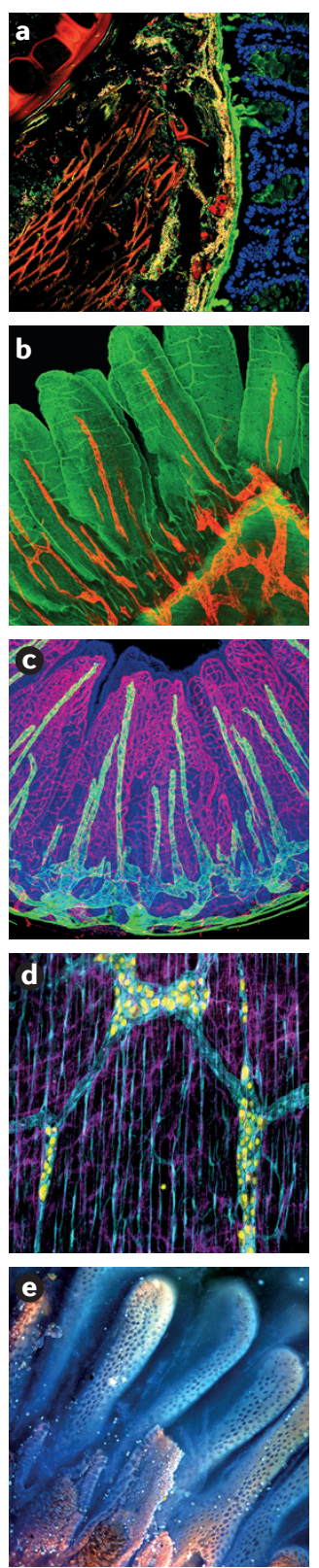

doi: 10.1038/nrgastro.2016.188 Published online 22 Dec 2016
Every year, Nature Reviews Gastroenterology \& Hepatology and the other clinical Nature Reviews journals run a competition to find an image to feature on our front covers for the next 12 months. We always receive some beautiful images, and this year was no exception. Once the competition closed there were $>60$ images to choose from, encompassing the breadth of gastroenterology and hepatology.

So what makes a great cover? First and foremost, the image and the research exemplified needs to be relevant to what we feature in the journal, showcasing an interesting aspect of gastroenterology or hepatology; for example, our 2016 covers featured a ductular reaction in the liver, a key process that occurs in many acute and chronic liver diseases. Next, it needs to be eye-catching: the image will grace our covers for a year and act as a 'window' into the journal. Finally, we have to think from an Art and Production point of view. Can we apply enough different colourways for 12 monthly issues? Is the resolution good enough, and does the subject fill the space nicely? After we have selected our winning image, our fantastic Production Editor, Jenna Johnston, works her magic to bring it to life, carefully tweaking the colours, brightness and contrast to make the image stand out whilst ensuring that we have 12 striking colourways for each month of the year.

The winning image on our front covers for 2017 (a) shows a mouse distal colon colonized by gut microbiota and was acquired using confocal microscopy. "The densely populated microbiota (yellow, red) and undigested plant fibre (red) travel down the lumen of the gut; the mucus (green) provides an essential interface between the host and bacteria, being both a carbohydrate-rich site of microbial attachment and an essential mechanical barrier," explains researcher Carolina Tropini (from the Sonnenburg group at Stanford University), who is interested in how physical perturbations affect the host and gut microbiota. The gut is a highly competitive environment and microscopy provides a snapshot into this diverse ecosystem and its complicated host-microbe interactions. The image was a unanimous favourite amongst the editorial team, not least because the gut microbiota is central to gastrointestinal health and disease but also because it matched all our criteria for a great cover image.

The high number of entries meant that the cover competition this year was particularly competitive and we wanted to showcase here some of our shortlisted favourites. Nicknamed "Steggy", owing to its likeness to Stegosaurus plates, the image submitted by Zbigniew Mikulski (b) shows a mouse small intestine, with the tissue structure highlighted in green and the lymphatic vessels in red. "Looking from top to bottom you can see epithelial cells on the villi, small holes that are openings of goblet cells; intestinal crypts are located between the villi and below them are two layers of muscles," says Mikulski. Keeping within the lymphatic theme, which is proving to be an emerging area in gastroenterology, another image considered for the cover, submitted by Shiue-Cheng (Tony) Tang (c), also highlighted the microstructure and vasculature of a mouse small intestine. "Although lymphatics play crucial roles in intestinal physiology and pathophysiology, the lymphatic vessel system is understudied because the intricate and dispersed network cannot be easily traced and studied," notes Tang. "The 3D vascular network presented in this image highlights our development of a new histological approach."

We had some superb images of the enteric nervous system for consideration, a subject that we often cover in the journal as neurogastroenterology is a fascinating and burgeoning area of research. One that caught our editorial eye (and invoked singing of "Purple Rain" by Prince because of the CMYK colouring) was by Meenakshi Rao (d). Here, confocal microscopy was used to depict the complex network and interactions of neurons, glia and interstitial cells of Cajal in the muscularis externa of the bowel wall. "Our laboratory studies the role of enteric glia in gastrointestinal biology as well as disease," says Rao. "This type of imaging allows us to visualize the diversity of glia as well as their functional interactions with other cell types in the gastrointestinal tract," she adds.

Our final shortlisted image by Richard Levenson (e) shows an advance in optical imaging techniques, using MUSE (microscopy with UV surface excitation) to provide a detailed view of human colon tissue. "MUSE takes advantage of the shallow penetration of the $280 \mathrm{~nm}$ UV light, which is used to excite fluorescent histological dyes," explains Levenson. "The combination permits the production of high-resolution images of tissues without requiring embedding or sectioning."

Thank you to everyone who submitted an image for consideration in our cover competition. Choosing our cover image is one of the highlights of the year and we are truly sorry to those we could not feature. The quality of submissions each year means that it is always a tough decision, but we are already looking forward to seeing the entries for our 2018 cover competition next year! 